\title{
Due significance need be given to pancreaticobiliary maljunction in pediatric practice
}

\begin{abstract}
Pancreaticobiliary maljunction (PBM) is a congenital anomaly defined as junction of the pancreatic and bile ducts located outside the duodenal wall beyond the influence of the sphincter of Oddi. There is PBM with biliary dilatation (choledochal cyst.CDC]) and PBM without biliary dilatation. In PBM, pancreaticobiliary reflux or biliopancreatic reflux are possible. PBM predisposes to cancer of biliary tract, pancreatitis and biliary stones. Manifestations, complications, diagnosis and treatment of PBM are highlighted in the background of recent scientific literature. The need of better awareness and search for PBM in pediatric practice is stressed.
\end{abstract}

Keywords: Duodenal, Sphincter, Reflux, Manifestations, Biliopancreatic ducts
Volume 7 Issue 4 - 2017

\author{
Rajendran Ramaswamy \\ Department of Pediatric Surgery, Maternity \& Children's \\ Hospital (under ministry of health), Saudi Arabia
}

Correspondence: Rajendran Ramaswamy, Consultant, Department of Pediatric Surgery, P.B.No:3600, Maternity \& Children's Hospital (under ministry of health), Najran, Saudi Arabia, Tel 966000000000 , Fax 0096675293915 , Email pedsurgdrraj57@yahoo.in

Received: December 21, 2017 | Published: December 27, 2017
Abbreviations: PBM, Pancreaticobiliary Maljunction; CDC, Choledochal Cyst; HCPBD, High Confluence of Pancreaticobiliary Ducts; CBDC, Common Bile Duct Carcinoma; IOC, Intraoperative Cholangiography; US, Ultrasonography; MRCP, Magnetic Resonance Cholangiopancreatography; MRI, Magnetic Resonance Imaging

\section{Introduction}

Pancreaticobiliary maljunction (PBM) is a congenital anomaly defined as junction of the pancreatic and bile ducts located outside the duodenal wall beyond the influence of the sphincter of Oddi, usually forming a markedly long common channel. Other names are "anomalous arrangement of pancreaticobiliary ducts" or "anomalous union of biliopancreatic ducts". ${ }^{1}$ Average length of common channel is $4.4 \mathrm{~mm} .{ }^{2}$ Some authors defined PBM as common channel more than $5 \mathrm{~mm}$.

PBM can be divided into PBM with biliary dilatation (choledochal cyst.CDC]) and PBM without biliary dilatation (maximal diameter of the bile duct $<$ or $=10 \mathrm{~mm}$ ). ${ }^{2,4}$ In addition, PBM can be classified into 3 distinct types based on imaging findings (Figure 1).

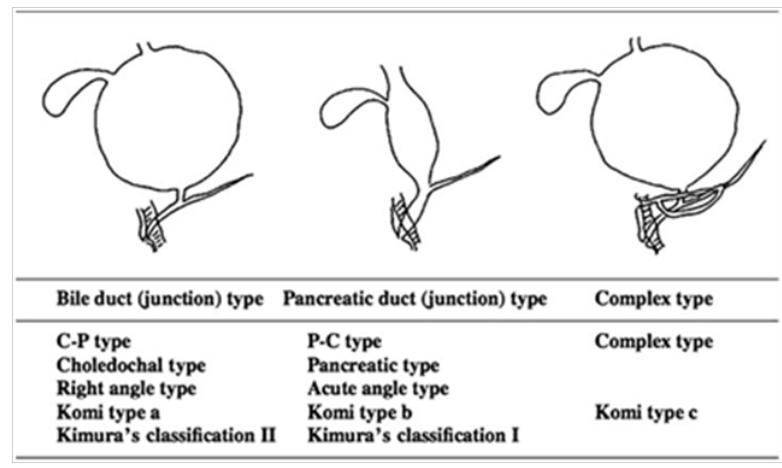

Figure I Pancreaticobiliary maljunction (PBM) classification. ${ }^{4}$

I. Bile duct (junction) type, in which the bile duct joins the pancreatic duct at right angle.

2. Pancreatic duct (junction) type, in which the pancreatic duct joins the bile duct at an acute angle

3. Complex type, in which the two ducts meet in a complex manner. ${ }^{1,4}$
PBM is often accompanied by pancreas divisum. The new Komi's classification added the presence/absence of the common channel dilatation and the concept of pancreas divisum to Komi's original classification. ${ }^{4,5}$ Liver and biliary tree develop from hepatic diverticulum of the foregut. The bilobed ventral pancreatic anlagen (cranial and caudal), develops from a part of the hepatic diverticulum that is close to its origin from foregut, and fuse at about the 4th week of gestation. PBM develops due to dysplasia of the ventral pancreas when it is formed, a process that is considered to affect the formation of hepatic diverticulum. The abnormal dilatation of the biliary tree occurs when the continuity between the hepatic diverticular epithelia and that of the primitive gut is lost. High confluence of pancreaticobiliary ducts (HCPBD) is a disease state in which the common channel is at least $6 \mathrm{~mm}$ long and the effect of the sphincter of Oddi does reach the pancreaticobiliary junction. ${ }^{4}$

The male-to-female ratio in congenital biliary dilatation is approximately $1: 3$, and it is especially predominant in young women. Its incidence is 1 in every 1,000 persons in Japan, 1 in 50,000-150,000 in western countries, and in 1 neonate in 2 million births. The most frequent subtypes of congenital biliary dilatation are:Todani type I (excluding type $\mathrm{Ib}$, which involves a segmental dilatation of CBD), in which duct dilation is limited to the common bile duct; and type $\mathrm{IV}-\mathrm{A}$, in which dilation of the intrahepatic bile duct(s) is added to the features of type I. In patients with congenital biliary dilatation with these two types the condition almost always is accompanied by PBM, while in the remaining types Ib, II, III, IV-B, or V, PBM is rarely seen. ${ }^{4,6}$ Practically the term "congenital biliary dilatation" is used instead of "PBM with biliary dilatation". ${ }^{4}$ Ono. ${ }^{7}$ f found that most pediatric cases reported as the nondilated type are slightly dilated (forme fruste) choledochal cysts and differ from the nondilated type seen in adults. Only $29 \%$ had a choledochal caliber within the normal range.

\section{Analysis of complications and their pathogenesis}

In PBM patients, pancreatic juices and bile reflux, mix, and accumulate, producing various pathological conditions in the biliary tract and pancreas. ${ }^{4}$ Since hydrostatic pressure within the pancreatic duct is usually higher than that in the common bile duct, pancreatic juice frequently refluxes into the bile duct (pancreatobiliary reflux). Refluxed proteolytic pancreatic enzymes and phospholipase A2 
are activated in the biliary tract and may cause inflammation and regeneration of epithelium of the biliary tract and induce metaplasia or promote cancer development and progression. Gall bladder mucosa of PBM patients can be considered premalignant. ${ }^{2} \mathrm{Li}$ et al. observed that the incidence of PBM was higher in common bile duct carcinoma (CBDC) patients, especially in relatively young patients; also the incidence of CDC was higher in CBDC patients with PBM. ${ }^{4,8-10}$ In a nationwide survey, biliary cancer was detected in $21.6 \%$ of adult patients with congenital biliary dilatation (bile duct cancer, $32.1 \%$ vs. gallbladder cancer, $62.3 \%$ ) and in $42.4 \%$ of PBM patients without biliary dilatation (bile duct cancer, $7.3 \%$ vs. gallbladder cancer, $88.1 \%) .4,9$ Nine cases of biliary tract cancer have been reported in children under 15years of age in Japan as a complication of PBM:7 bile duct cancers and 2 gallbladder cancers. ${ }^{4}$ Pancreatobiliary reflux and premalignant changes in the gallbladder can occur in patients with a relatively long common channel of HCPBD also. ${ }^{2}$

Patients with PBM are more predisposed to forming biliary tract stones than individuals without this disease. Biliary tract stones are found in $17.9 \%$ of patients with congenital biliary dilatation and in $27.3 \%$ of patients suffering from PBM without biliary dilatation. Gallstones in the bile duct are most frequently found in patients with biliary dilatation, and gallstones in the gallbladder are most frequent in patients with PBM without biliary dilatation.4 In recent years, there is an increase in the incidence of nonhaemolitic cholilithiasis in childhood; PBM should be searched as an underlying cause. Bile may reflux into the pancreatic duct via PBM in some situations, such as with cholangitis or bile stasis in the biliary tract. Refluxed bile may activate pancreatic enzymes, particularly phospholipase A2, and may thus cause acute pancreatitis. Acute pancreatitis, occurs in $9 \%$ of adult PBM-patients and in $28 \%-43.6 \%$ of children. Dilatation of the common channel, pancreatic duct dilatation, complicated anomalies in the pancreatic duct in the pancreas head, and protein plugs are the causes of the acute pancreatitis associated with PBM. The frequency of acute pancreatitis in children with choledochal cyst is reportedly as high as $68 \%$. Acute pancreatitis associated with PBM is usually mild but recurrent. Chronic pancreatitis also is possible in individuals with PBM. ${ }^{4}$ The clinical features of HCPBD are similar to those observed in PBM, including pancreatobiliary reflux and biliopancreatic reflux. ${ }^{4}$

\section{What clinical manifestations are associated with PBM?}

PBM with congenital biliary dilatation of neonatal/ infant onset patients have jaundice and abdominal mass while those with onset during early childhood manifest mainly with abdominal pain. ${ }^{4}$ Children with PBM without choledochal cysts usually do not show any symptoms except for abdominal pain and hyperamylasemia. ${ }^{1}$

\section{Pancreaticobiliary maljunction is diagnosed}

Mainly by endoscopic retrograde cholangiopancreatography; however, in pediatric patients, it may be diagnosed by intraoperative cholangiography (IOC), and more recently has been diagnosed by computed tomographic cholangiography or contrast-enhanced helical computed tomography. ${ }^{1}$ Serum levels of amylase, bilirubin, and biliary enzymes may become abnormal when the patient becomes symptomatic. ${ }^{4}$ Ultrasonography (US) is useful to screen for PBM. Dilatation of the common bile duct and/or intrahepatic bile duct by US diagnoses congenital biliary dilatation. A thickening of the hypoechoic inner layer of the gall bladder wall may be fundamental for the diagnosis of PBM without biliary dilatation. ${ }^{4}$ Magnetic resonance cholangiopancreatography (MRCP) detects PBM 82-100 $\%$ in adults and $40-80 \%$ in children. ${ }^{4}$ Endoscopic US can confirm the status of the pancreaticobiliary junction outside the duodenal wall. ${ }^{4}$
It is possible to diagnose congenital biliary dilatation prenatally by fetal USS or magnetic resonance imaging (MRI) when the bile duct is dilated in a cystic form. ${ }^{4}$ To detect PBM without biliary dilatation early, MRCP is recommended for patients showing gallbladder wall thickening on screening US. ${ }^{9}$ Pancreaticobiliary reflux in PBM patients can be visualized radiologically using secretin-stimulated dynamic MRCP. In a retrospective study of imaging with various modalities in 75 pediatric patients, Guo. ${ }^{3}$ ] concluded that US, IOC, $\mathrm{CT}$ and MRCP are valuable in showing dilatation of the bile duct and complications in pediatric PBM. MRCP is nonnvasive, gives clear views of the pancreaticobiliary junction and should be the first choice for the diagnosis of PBM in children. ${ }^{3,6}$

Once the diagnosis of PBM is established, immediate prophylactic surgery is recommended irrespective of the presence or absence of symptoms. ${ }^{9}$ Symptoms become evident and treatment for PBM is received in $53.7 \%$ of children by 3 years of age and in $91.7 \%$ by the age of $6 .{ }^{4,9}$ The treatment of choice for PBM with biliary dilatation is prophylactic flow-diversion surgery (bile duct resection and hepaticodochojejunostomy) before malignant changes can take place in the biliary tract. ${ }^{2,10}$ On the other hand, treatment of PBM without biliary dilatation or cancer is controversial. Prophylactic cholecystectomy is alone performed in many institutions, as most biliary cancers that develop in PBM patients without biliary dilatation are gallbladder cancers. ${ }^{2,4}$ However, some surgeons suggest that the extra hepatic bile duct together with gallbladder should be excised, because of a perceived risk of bile duct cancer. ${ }^{2,9}$ Symptomatic neonates and infants should be operated on as soon as possible, whereas for asymptomatic cases, elective operations at around 3-6 months of age may be considered while their main organ function, such as that of the liver, is monitored carefully. ${ }^{4}$ IOC is essential to confirm the relative stenosis of the hepatic hilar bile duct, to check for protein plugs, and to define the site of bile duct resection for biliary tract reconstruction. The bile duct on the pancreatic side should be resected just above its junction with the pancreatic duct. ${ }^{4}$ This and removal of stenoses of the hepatic ducts are necessary to prevent serious complications after surgery. ${ }^{10}$

In conclusion, better awareness about PBM, its complications, diagnosis and treatment in childhood can prevent serious consequences in adulthood.

\section{Acknowledgments}

None.

\section{Conflict of interest}

There is no conflict of interest.

\section{References}

1. Ono S, Fumino S, Iwai N. Diagnosis and treatment of pancreaticobiliary maljunction in children. Surg Today. 2011;41(5):601-605.

2. Kamisawa T, Takuma K, Anjiki H, et al. Pancreaticobiliary maljunction. Clin Gastroenterol Hepatol. 2009;7(11 Suppl):S84-S88.

3. Guo W, Huang S, Wang J. Imaging findings in 75 pediatric patients with pancreaticobiliary maljunction:a retrospective case study. Pediatr Surg Int. 2012;28(10):983-988.

4. Kamisawa T, Ando H, Suyama M, et al. Working Committee of Clinical Practice Guidelines for Pancreaticobiliary Maljunction. Japanese clinical practice guidelines for pancreaticobiliary maljunction. J Gastroenterol. 2012;47:731-759 
5. Komi N. New classification of anomalous arrangement of the pancreaticobiliary ducts (APBD) in choledochal cyst:a proposal of new Komi's classification of APBD (in Japanese). J Pancr Soc. 1991;6(3):234-244.

6. Ankur A, Amar M, Shalini T, Seema A. Anomalous Pancreaticobiliary Junction. JPGN. 2014;58(2):e13.

7. Ono Y, Kaneko K, Tainaka T. Pancreaticobiliary Maljunction Without Bile Duct Dilatation in Children:Distinction From Choledochal Cyst. JPGN. 2018;46(5):555-560.
8. Li Y, Wei J, Zhao Z. Pancreaticobiliary Maljunction Is Associated with Common Bile Duct Carcinoma:A Meta-Analysis. The Scientific World Journal, Article ID 618670, p.9.

9. Kamisawa T, Kuruma S, Tabata T, et al. Pancreaticobiliary maljunction and biliary cancer. J Gastroenterol. 2015;50(3):273-279.

10. Kamisawa T, Kaneko K, Itoi T. Pancreaticobiliary maljunction and congenital biliary dilatation. Lancet Gastroenterol Hepatol. 2017;2(8):610-618. 